\title{
Schistosoma haematobium and soil- transmitted Helminths in Tana Delta District of Kenya: infection and morbidity patterns in primary schoolchildren from two isolated villages
}

Kariuki H. Njaanake ${ }^{1 *}$, Birgitte J. Vennervald ${ }^{2}$, Paul E. Simonsen², Henry Madsen², Dunstan A. Mukoko³, Gachuhi Kimani ${ }^{4}$, Walter G. Jaoko ${ }^{1}$ and Benson B. Estambale ${ }^{5}$

\begin{abstract}
Background: Schistosomes and soil-transmitted helminths (STH) (hookworm, Trichuris trichiura and Ascaris lumbricoides) are widely distributed in developing countries where they infect over 230 million and 1.5 billion people, respectively. The parasites are frequently co-endemic and many individuals are co-infected with two or more of the species, but information on how the parasites interact in co-infected individuals is scarce. The present study assessed Schistosoma haematobium and STH infection and morbidity patterns among school children in a hyper-endemic focus in the Tana River delta of coastal Kenya.

Methods: Two hundred and sixty-two children aged 5-12 years from two primary schools were enrolled in the study. For each child, urine was examined for S. haematobium eggs and haematuria, stool was examined for STH eggs, peripheral blood was examined for eosinophilia and haemoglobin level, the urinary tract was ultrasoundexamined for S. haematobium-related pathology, and the height and weight was measured and used to calculate the body mass index (BMI).

Results: Prevalences of S. haematobium, hookworm, T. trichiura and A. lumbricoides infection were 94, 81, 88 and $46 \%$, respectively. There was no significant association between S. haematobium and STH infection but intensity of hookworm infection significantly increased with that of $T$. trichiura. Lower BMI scores were associated with high intensity of $S$. haematobium (difference $=-0.48, p>0.05$ ) and A. lumbricoides (difference $=-0.67, p<0.05$ ). Haematuria (both macro and micro) was common and associated with S. haematobium infection, while anaemia was associated with high intensity of $S$. haematobium $(\mathrm{OR}=2.08, p<0.05)$ and high hookworm infections $\mathrm{OR}=4.75$; $p<0.001)$. The majority of children had eosinophilia, which was significantly associated with high intensity of hookworm infection $(\mathrm{OR}=5.34, p<0.05)$. Overall $38 \%$ of the children had ultrasound-detectable urinary tract morbidity, which was associated with high intensity of $S$. haematobium infection $(\mathrm{OR}=3.13, p<0.05)$.

Conclusion: Prevalences of S. haematobium and STH infections among the primary school children were high and the parasites were responsible for significant morbidity. A clear synergistic interaction was observed between hookworm and T. trichiura infections. Increased coverage in administration of praziquantel and albendazole in the area is recommended to control morbidity due to these infections.
\end{abstract}

Keywords: Schistosoma haematobium, Soil-transmitted helminths, Infections, Morbidity patterns

\footnotetext{
*Correspondence: kn@uonbi.ac.ke

'Department of Medical Microbiology, College of Health Sciences, University

of Nairobi, P.O. Box 19676-00202, Nairobi, Kenya

Full list of author information is available at the end of the article
} 


\section{Background}

Schistosomiasis and soil-transmitted helminthiases are important neglected tropical diseases. It is estimated that schistosomes infect over 230 million people worldwide whereas soil-transmitted helminths (STH) such as hookworms, Trichuris trichiura and Ascaris lumbricoides infect over 1.5 billion resulting in 1.7 million and 5.2 million disability-adjusted life years, respectively [1-3]. SubSaharan Africa bears the largest burden of these infections, with primary school aged children from resource-poor communities being the most highly affected $[3,4]$.

Due to geographical overlap of transmission foci many individuals are co-infected with Schistosoma spp. and one or more of the STH species $[5,6]$. However, marked heterogeneity in pattern of prevalence and co-infection exist [7-9]. Socioeconomic status, human behaviour, environmental factors and demography may be partly responsible for this [10]. Parasite-parasite and parasitehost interactions may also affect infection patterns in ways that are not yet clearly understood [11-14]. Increasing evidence moreover shows that such interactions may lead to not only increased worm burdens but increased morbidity as well [15].

Kenya is endemic for S. haematobium, S. mansoni and $\mathrm{STH}$ with foci of transmission scattered all over the country [9]. Existing data indicate that there is considerable intra-country spatial heterogeneity in prevalence of these infections with the coastal strip being one of the largest foci for S. haematobium and STH transmission in the country [7, 9]. Several studies on S. haematobium have been carried out in the southern and middle parts of the coastal strip, spanning from Kwale county in the south to Malindi county in the north [16-21]. Similarly, STH infections have been studied elsewhere in the coastal region [22-24]. There are, however, no comprehensive and systematic studies on schistosomiasis and $\mathrm{STH}$ infections in the northern part of coastal Kenya, which comprise an important endemic focus [9].

We conducted a study to document the pattern of $S$. haematobium and STH infections and resultant morbidity among schoolchildren in two isolated villages of Tana Delta District, coastal Kenya.

\section{Methods}

\section{Study area}

We chose two primary schools located in two isolated villages from where there had been no previous studies or treatment campaigns for schistosomiasis and STH: Kau in Kipini Division ( $\left.2^{\circ} 29^{\prime} 20^{\prime \prime} \mathrm{S}, 40^{\circ} 27^{\prime} 7^{\prime \prime} \mathrm{E}\right)$ and Ozi in Garsen South Division (2 $31^{\prime} 9^{\prime \prime} \mathrm{S}, 40^{\circ} 27^{\prime} 40^{\prime \prime} \mathrm{E}$ ) in Tana Delta District, on the north coast of Kenya. Both villages are located on the banks of River Tana, about $5 \mathrm{~km}$ apart. The area is prone to seasonal flooding and, during the dry season, numerous swamps are scattered all over the area. The residents practice rice farming in the flood plains in the delta of River Tana as well as fishing in the river and swamps. Within each village, there is one primary school and children from each village attend their respective school. School enrolment in the two villages was high (about $90 \%$ ) but absenteeism was also high.

\section{Study design}

This was a cross-sectional study of prevalence, intensity and morbidity due to S. haematobium and STH infections among schoolchildren aged between 5 and 12 years in the two villages. Three urine and stool samples were collected from each study participant (each urine and stool sample being collected on a different day), and examined for S. haematobium and STH eggs, respectively. One $2 \mathrm{ml}$ venous blood sample was collected using a syringe and gauge 23 needle. Part of the blood $(20 \mu \mathrm{l})$ was used to prepare a thin blood smear for malaria parasite examination and differential white blood cell count. Another $10 \mu \mathrm{l}$ was used for haemoglobin level estimation. The rest was used for serum preparation for another study [25]. Weight and height of each child was recorded. Finally, their urinary tract was examined for $S$. haematobium-related morbidity using ultrasound. Prior to the start of the study, ethical approval was obtained from the University of Nairobi - Kenyatta National Hospital Ethics and Research Committee in Kenya (Approval No. P91/3/2009). Written informed consent for participation was obtained from each child's parent or guardian. After the field sample collection, all children were treated with praziquantel $(40 \mathrm{mg} / \mathrm{kg})$ and albendazole (400 mg) in accordance with the national guidelines. Those who had not cleared the infections after the initial treatment were re-treated appropriately. Those presenting with other minor clinical manifestations including anemia were offered free treatment from the study team and those with clinical manifestations beyond the ability of the team were referred to the local health centre for medical attention.

\section{Urine examination for S. haematobium eggs}

Ten millilitre of each of three consecutive urine samples from each child was filtered through a polycarbonate filter $(12 \mu \mathrm{m}$ pore-size; GE Water \& Process Technologies Inc., USA) and the filter was examined microscopically for S. haematobium eggs [26]. The results, based on arithmetic mean of the three egg counts from each child, were classified into negative, light (1-49 eggs/10 ml urine) or heavy infections ( $\geq 50$ eggs/10 ml urine) according to WHO $[27,28]$.

\section{Stool examination for soil transmitted helminth eggs}

From each of the three stool samples obtained from each child, $41.7 \mathrm{mg}$ was used to prepare a slide using standard 
Kato-Katz technique and was examined microscopically for the presence of hookworm, T. trichiura and A. lumbricoides eggs within $1 \mathrm{~h}$ of preparation [29]. Individual infections with $\mathrm{STH}$ were classified into three groups based on a mean of the three egg counts according to WHO classification [27, 28]. Infections with hookworm were classified as negative, light (1-1,999 eggs/g stool) and moderate to heavy ( $\geq 2,000 \mathrm{eggs} / \mathrm{g}$ stool). Infections with $T$. trichiura were classified as negative, light (1-999 eggs/g stool) and moderate to heavy $(\geq 1,000$ eggs/g stool) whereas those of $A$. lumbricoides were classified as negative, light (1-4,999 eggs/g stool) and moderate to heavy ( $\geq 5,000$ eggs/g stool).

\section{Urine examination for haematuria}

At the time of recruitment, participants were asked whether they had seen blood in their urine and during urine sample collection, each sample was examined visually for the presence of blood (macro-haematuria). The samples were also tested for presence or absence of occult blood (micro-haematuria) with dipstix (URISCAN ${ }^{\oplus}$, YD Diagnostics, Korea) according to the manufacturer's instructions. Change in colour of the test pad was noted within $1 \mathrm{~min}$ and interpreted according to a chart provided by the manufacturer.

\section{Haemoglobin}

About $10 \mu \mathrm{l}$ of venous blood was used to estimate haemoglobin concentration using a portable haemoglobinometer, which gives readings in $\mathrm{g} / \mathrm{dl}$, according to manufacturer's instructions (HemoCue Hb 301, HemoCue $^{\curvearrowleft}$, Sweden). A new standard cuvette supplied together with the haemoglobinometer was used every day for quality control. Haemoglobin concentrations were expressed in $\mathrm{g} / \mathrm{dl}$ and the results were recorded and categorised into two: normal ( $\geq 11.5 \mathrm{~g} / \mathrm{dl}$ of blood) or anaemia $(<11.5 \mathrm{~g} / \mathrm{dl}$ of blood) [30].

\section{Blood cell count}

A thin smear blood film was prepared using a small amount of each venous blood sample from each child and stained with Giemsa [26]. Differential cell count was performed on 100 white blood cells in each film and the numbers of each type of white blood cells expressed as a percentage of the total 100 white blood cells. Eosinophilia was defined as eosinophils above $7 \%$ of white blood cells on the blood film (http://emedicine.medscape.com/article/ 2085133-overview\#a1; Accessed 2nd June 2015).

\section{Anthropometry}

The weight of each participant was measured to the nearest $0.5 \mathrm{~kg}$ using an electronic scale (Salter Electronic ${ }^{\odot}$ ), with the participant wearing only light clothes and no shoes. Height was measured to the nearest $1 \mathrm{~cm}$ using a portable stadiometer with the participants not wearing shoes. Body mass index (BMI) was calculated as weight $(\mathrm{kg}) /(\text { height }(\mathrm{m}))^{2}$ and categorised into two: normal BMI $\left(\geq 18.5 \mathrm{~kg} / \mathrm{m}^{2}\right)$ or low BMI $\left(<18.5 \mathrm{~kg} / \mathrm{m}^{2}\right)$ [31].

\section{Ultrasound}

Ultrasound examination of the urinary tracts of each child was performed by an experienced ultrasonographer using a portable convex sector scanner (SSD-500; Aloka, Tokyo, Japan) according to the Niamey protocol [32] and bladder, ureter and kidney pathology was recorded. If any kidney and/ or ureter dilatation was observed the child was asked to empty the bladder and come back for re-examination.

\section{Statistical analyses}

S. haematobium and STH egg counts were summed over the three samples, or for some just two samples, per child, and the amount of urine or faeces examined was used as an offset in the analyses of infection intensity. For graphical representation, egg counts were expressed as number of eggs per $10 \mathrm{ml}$ urine (S. haematobium) or as eggs per $\mathrm{g}$ faeces (STH infections) and mean intensity and $95 \%$ confidence limits were based on the average and $95 \%$ confidence limits of $\log (x+1)$ back transformed to the original scale. Since the recommended categories for intensity of infection [27, 28] produced highly uneven sample sizes for the different groups, we decided to cut intensity (from 1 to the maximum egg count) of each infection into three groups (low, medium and high) with approximately equal sample size. Zero counts (un-infected) constituted the base group against which the other three categories were compared. For $S$. haematobium, however, only 18 children were not infected and therefore this group was merged with the low intensity category.

Intensity of infection (as original counts) for each of the 4 infections was analyzed using negative binomial regression [33] in a generalized linear model with a log link function and with school, gender, age group and categories of infection intensity of the other three parasites as predictors. These predictors were all tested individually and later jointly in multi-variable models where initially all variables were entered and non-significant predictors (including non-significant levels of intensity of infection starting from the lowest category) were manually removed until only significant predictors were retained in the final model. Testing for over dispersion was based on the chi-square statistics [33]. Interactions between parasite intensity of infection (categories) was done pair-wise when main effects were significant without the interaction.

Similarly, prevalence of infection (not-infected/infected) for each infection separately was analyzed using logistic 
regression analysis [34] specified in a generalized linear model with a logit link function and otherwise following the same principle of modelling as above. Also for morbidity indicators (except body mass index), logistic regression analysis was done following the above guidelines including testing interactions between parasite species. BMI was analysed in a generalized linear model of the Gaussian family and with unit link function. For missing records of morbidity indicators, we always tested whether there was any bias between children sampled and those who were not in relation to the demographic variables and infections, but this was not the case. P-values less than 0.05 were taken to indicate significant effects.

\section{Results}

\section{Study population and general infection levels}

In total, 262 children with an average age of 9.7 years were recruited in the study. There were 128 boys and 134 girls and mean age in the two sexes was similar (9.9 vs. 9.6 years, respectively). A total of 96 children were aged between 5 and 9 years, and the other 166 children between 10 and 12 years. Of the 262 enrolled children, 242 provided three urine samples, each sample on a different day. The remaining 20 children provided two urine samples. All those who provided two samples only were positive for S. haematobium eggs. Two hundred and twenty-nine children provided three stool samples, each on a different day, whereas the other 33 provided two stool samples. Of those who provided two stool samples, 7 tested negative for STH eggs. All 262 children were included in the analyses.

The overall prevalence of S. haematobium was $94 \%$, with $38 \%$ of the children having light (following WHO classification) and $56 \%$ heavy infections; $81 \%$ were infected with hookworm (75\% light and $6 \%$ moderate to heavy), $88 \%$ with $T$. trichiura ( $81 \%$ light and $7 \%$ moderate to heavy) and $45 \%$ with $A$. lumbricoides (43\% light and $3 \%$ moderate to heavy). The prevalence and intensity of $S$. haematobium and STH infections by school and age group are shown in Fig. 1.

\section{Analysis of infection prevalence and intensity}

Prevalence and intensity of each of the four infections was first analysed separately using school, gender, age group and category of intensity of each of the other three infections as predictors (results shown in Table 1). Multivariable analyses were thereafter performed and the results of these are presented as follows.

For prevalence of $S$. haematobium infection, only age group remained significant, i.e., odds of infection in the $10-12$ years age group was $4.17(p<0.05)$ of that in the $5-9$ years age group. The final model for hookworm infection showed that school $(\mathrm{OR}=3.10, p<0.01)$, gender (3.13, $p<0.01)$ and T. trichiura infection $(3.08, p<0.01$,

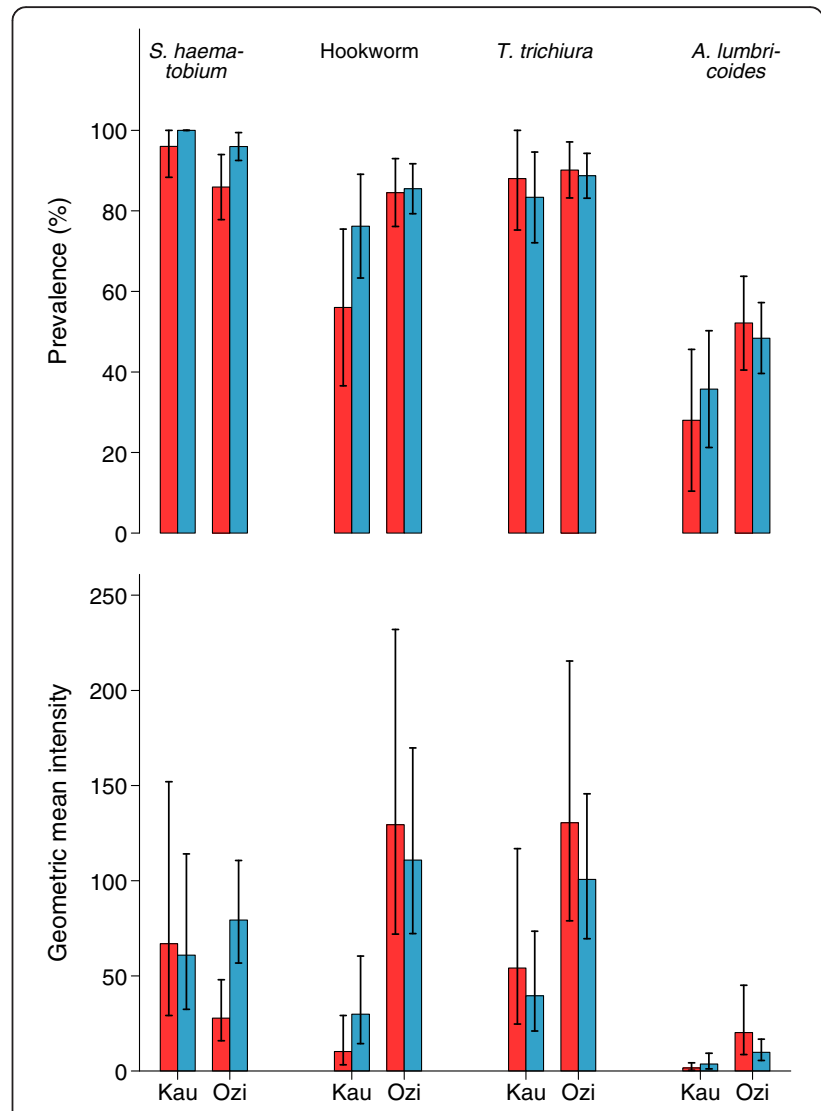

Fig. 1 Prevalence (\%) and geometric mean intensity of infection by school (Kau and Ozi) and age group. Red bars $=5-9$ years and blue bars $=10-12$ years. Geometric mean intensity given in no. of eggs per $10 \mathrm{ml}$ urine (Schistosoma haematobium) or per $\mathrm{g}$ faeces (Trichuris trichiura, hookworm, and Ascaris lumbricoides). Error bars indicate $95 \%$ confidence interval. Note that all 10-12 years old children were infected with S. haematobium; hence no error bar

respectively) were associated with higher odds of infection. The ORs for the three categories of T. trichiura infection intensity did not differ significantly and they were therefore pooled. Odds of $T$. trichiura infection was higher among those with medium and high hookworm infection intensity, i.e., odds of infection in children with medium intensity was $9.54(\mathrm{p}<0.01)$ and in those with high intensity was $4.50(p<0.01)$ of that of children with no or low hookworm infection. For $A$. lumbricoides infection only high intensity of both hookworm $(\mathrm{OR}=2.36, p<0.01)$ and T. trichiura $(2.18, p<0.01)$ remained in the model. The interaction term between these two infections was not significant.

Intensity of $S$. haematobium infection did not show association with any of the predictors. Intensity of hookworm infection showed that school $(\mathrm{CR}=4.17, p<0.001)$ and gender $(\mathrm{CR}=2.29, p<0.001)$ were significant. Also in this model, medium and high intensity of $T$. trichiura infection were associated with higher intensity of hookworm infection, i.e., $\mathrm{CR}=1.74(p<0.01)$ and $2.22(p<0.001)$, 
Table 1 Association of Schistosoma haematobium and STH infections in the school children with various predictors. Coefficents are exponentiated regression coefficients from logistic regression (infection status) or from negative binomial regression (intensity of infection) as compared to the reference category, i.e., Kau school for school, girls for gender, 5-9 years for age class, non-infected plus light infection for $S$. haematobium and uninfected for the three other infections. Results from the multivariable analyses are $p$ resented in the text

\begin{tabular}{|c|c|c|c|c|c|c|c|c|c|}
\hline & & Infection status (c & dds ratio) & & & Intensity of infect & ion (count ra & & \\
\hline & N & S. haematobium & Hookworm & T. trichiura & A. lumbricoides & S. haematobium & Hookworm & T. trichiura & A. lumbricoides \\
\hline Ozi school & 195 & 0.18 & $2.61^{* *}$ & 1.45 & $2.02^{*}$ & 0.90 & $4.12^{* * *}$ & $3.65^{* * *}$ & 2.44 \\
\hline Boys & 128 & 1.64 & $2.66^{* *}$ & 1.37 & 1.35 & 1.22 & $1.97^{*}$ & $0.51^{* * *}$ & 1.53 \\
\hline Age $10-12$ years & 166 & $4.17^{*}$ & 1.47 & 0.80 & 0.97 & 1.34 & 0.90 & $0.50^{* * *}$ & 0.62 \\
\hline S. haematobium & & & & & & & & & \\
\hline Medium: 35-231 & 82 & - & 0.98 & 1.41 & 1.21 & - & 1.67 & $0.46^{* * *}$ & 0.66 \\
\hline High: 232-1000 & 82 & - & 1.36 & 1.10 & 1.15 & - & 1.22 & $0.50^{* *}$ & 0.97 \\
\hline Hookworm & & & & & & & & & \\
\hline Low: 7-106 & 68 & 0.90 & - & 1.34 & 0.58 & 0.98 & - & 0.87 & 0.61 \\
\hline Medium: 113-453 & 73 & 0.47 & - & $11.21^{* *}$ & 1.38 & 1.19 & - & $3.95^{* * *}$ & $7.58^{* * *}$ \\
\hline High: 460-3786 & 71 & 0.55 & - & $5.29^{* *}$ & 2.59 & 1.14 & - & $3.35^{* * *}$ & $3.70^{*}$ \\
\hline T. trichiura & & & & & & & & & \\
\hline Low:7-85 & 77 & 1.25 & 2.23 & - & 1.96 & 1.01 & $2.37^{*}$ & - & 0.82 \\
\hline Medium: 92-290 & 76 & 0.60 & $3.70^{*}$ & - & $3.09^{*}$ & 0.91 & $3.20^{* *}$ & - & 2.19 \\
\hline High: 297-21231 & 78 & 0.26 & $4.84^{* *}$ & - & $5.49^{* * *}$ & 1.00 & $4.96^{* * *}$ & - & $12.00^{* * *}$ \\
\hline A. lumbricoides & & & & & & & & & \\
\hline Low: 7-43 & 38 & 0.38 & 0.74 & 2.35 & - & 1.12 & $1.96^{*}$ & 1.48 & - \\
\hline Medium: 50-297 & 41 & 0.55 & 1.55 & 2.55 & - & 1.15 & $1.85^{*}$ & $2.14^{* *}$ & - \\
\hline High: 311-18789 & 40 & 0.53 & 2.39 & $7.87^{*}$ & - & 0.97 & $2.29^{* *}$ & $5.67^{* * *}$ & - \\
\hline
\end{tabular}

${ }^{*} p<0.05 ;{ }^{* *} p<0.01 ;{ }^{* * *} p<0.001$

respectively. The dispersion statistics was 1.05 , indicating a slight over-dispersion.

T. trichiura intensity was significantly associated with school $(\mathrm{CR}=1.61, p<0.001)$, gender $(\mathrm{CR}=0.65, p<0.01)$, and medium $(\mathrm{CR}=2.37,<0.001)$ and high hookworm intensity of infection $(\mathrm{CR}=3.49,<0.001)$. The $A$. lumbricoides egg count categories from negative to medium were combined and there was a significant interaction $(p<0.001)$ between this variable and S. haematobium egg count categories. The main effects of S. haematobium (i.e., the effect of $S$. haematobium intensity in children who had no to medium level of $A$. lumbricoides infection) was not significant, i.e., $(\mathrm{CR}=0.79, p>0.05)$ and $(\mathrm{CR}=0.93, p>0.05)$ for medium and high $S$. haematobium egg counts, respectively, while for children with high A. lumbricoides egg counts, $T$. trichiura counts were significantly lower when they also had medium $(\mathrm{CR}=0.24$, $p<0.01)$ or high $(\mathrm{CR}=0.15, p<0.001)$ S. haematobium egg counts. These last two coefficients were not significantly different. The main effect of $A$. lumbricoides (i.e., in children who had no or light $S$. haematobium infection) was $\mathrm{CR}=6.50(p<0.001)$. The model without the interaction was over dispersed (dispersion statistics $=1.41$ ), but although inclusion of the interaction improved the model (dispersion statistics $=1.14$ ), the model was somewhat over dispersed, so coefficients should be interpreted with care.

High $A$. lumbricoides intensity was significantly associated with gender $(\mathrm{CR}=5.98, p<0.001)$ and the $T$. trichiura intensity categories medium $(\mathrm{CR}=4.74, p<0.01)$ and high $(C R=39.53, p<0.001)$. The dispersion statistics (1.20) indicated over-dispersion.

\section{Analysis of morbidity indicators}

Associations between the various morbidity indicators and the various predictors individually are summarized in Table 2.

Weight and height measurements were available for 231 children. There was however no significant difference between the 231 and the remaining 31 children whose weight and height measures were not available in terms of infection prevalence and intensity. The great majority $(95 \%)$ of children were underweight $(\mathrm{BMI}<11)$ and this created a problem for the statistical evaluation of the impact of infections. Therefore, we instead used the BMI scores directly. Higher BMI scores were associated with age and low hookworm infection intensity, while lower scores were associated with high intensity of S. haematobium and A. lumbricoides infection (Table 2). 
Table 2 Association of morbidity indicators in the school children with and various predictors. The coefficients reflect either mean difference (BMI) or odds ratios from logistic regression analysis between a given factor level and its corresponding baseline group. Results from the multivariable analyses are presented in the text

\begin{tabular}{|c|c|c|c|c|c|c|c|c|c|}
\hline & $\begin{array}{l}\text { BMI } \\
(n=231)\end{array}$ & Anaemia & $\begin{array}{l}\text { Reported blood } \\
\text { in urine }\end{array}$ & $\begin{array}{l}\text { Macro- } \\
\text { haematuria }\end{array}$ & $\begin{array}{l}\text { Micro- } \\
\text { haematuria }\end{array}$ & Eosinophilia & $\begin{array}{l}\text { Bladder } \\
\text { pathology }\end{array}$ & $\begin{array}{l}\text { Upper urinary } \\
\text { tract pathology }\end{array}$ & $\begin{array}{l}\text { Total } \\
\text { pathology }\end{array}$ \\
\hline No. examined/no. affected & - & $230 / 141$ & $211 / 155$ & $262 / 165$ & $262 / 181$ & $217 / 201$ & $219 / 69$ & $219 / 23$ & $219 / 83$ \\
\hline Ozi school & -0.34 & 1.70 & 1.53 & 0.65 & 0.85 & 1.24 & 1.15 & 0.64 & 0.82 \\
\hline Boys & 0.11 & 1.03 & 0.91 & $2.14^{* *}$ & 1.61 & 2.05 & 1.52 & 1.21 & 1.50 \\
\hline Age $10-12$ years & $0.84^{* * *}$ & $0.54^{*}$ & $1.87^{* *}$ & $1.80^{* *}$ & $1.87^{*}$ & 2.19 & 1.24 & 2.09 & 1.44 \\
\hline \multicolumn{10}{|l|}{ S. haematobium } \\
\hline Medium: 35-231 & 0.12 & 1.87 & $4.67^{* * *}$ & $10.71^{* * *}$ & $16.13^{* * *}$ & 1.34 & $2.85^{* *}$ & 0.58 & $2.17^{*}$ \\
\hline High: 232-1000 & -0.48 & $2.08^{*}$ & $4.32^{* * *}$ & $279.82^{* * *}$ & ne & 2.34 & $4.40^{* * *}$ & $3.13^{*}$ & $4.40^{* * *}$ \\
\hline \multicolumn{10}{|l|}{ Hookworm } \\
\hline Low:7-106 & $0.76^{*}$ & 1.17 & $0.29 *$ & 1.19 & 1.72 & 2.26 & 0.99 & 0.97 & 0.84 \\
\hline Medium: 113-453 & 0.23 & 1.60 & 0.40 & 1.34 & 1.65 & 3.44 & 1.85 & 0.90 & 1.63 \\
\hline High: 460-3786 & -0.06 & $4.75^{* * *}$ & $0.32^{*}$ & 1.87 & 1.59 & $5.34^{*}$ & 1.35 & 1.53 & 1.42 \\
\hline \multicolumn{10}{|l|}{ T. trichiura } \\
\hline Low:7-85 & 0.46 & 1.24 & 0.44 & 1.38 & 1.23 & 0.42 & 0.67 & 1.31 & 0.75 \\
\hline Medium: 92-290 & 0.68 & 1.80 & 1.01 & 0.80 & 0.53 & 0.61 & 0.78 & 0.16 & 0.70 \\
\hline High: 297-21231 & 0.51 & 1.63 & 0.50 & 0.75 & 0.70 & 0.56 & 0.62 & 2.53 & 0.88 \\
\hline \multicolumn{10}{|l|}{ A. lumbricoides } \\
\hline Low: 7-43 & -0.03 & 1.21 & 0.95 & 1.96 & 1.51 & 0.52 & 0.95 & 0.24 & 0.83 \\
\hline Medium: 50-297 & -0.08 & 1.55 & 0.46 & 0.86 & $0.47^{*}$ & 1.15 & 1.41 & 0.78 & 1.22 \\
\hline High: 311-18789 & $-0.67^{*}$ & 1.21 & 0.78 & 0.82 & 0.83 & 1.05 & 0.99 & 1.79 & 1.45 \\
\hline
\end{tabular}

${ }^{*} p<0.05 ;{ }^{* *} p<0.01 ;{ }^{* * *} p<0.001$

Age $(b=0.82, p<0.001)$, low hookworm $(b=0.63$, $p<0.01)$ and high intensity of $S$. haematobium infection $(\mathrm{b}=-0.57, p<0.01)$ remained in multivariable model. The interaction between hookworm and S. haematobium infections was not significant.

Venous blood samples from 230 children were tested for haemoglobin levels. The overall prevalence of anaemia was $61.3 \%$. In multi-variable analysis, anaemia was associated with age group $(\mathrm{OR}=0.47, p<0.05)$, medium $(\mathrm{OR}=2.25, p<0.01)$ and high intensity $(\mathrm{OR}=2.50, p<0.01)$ of $S$. haematobium infection and high intensity of hookworm infection $(\mathrm{OR}=3.74, p<0.001)$.

A total of 211 children replied to whether they had seen blood in their urine in the past 3 months. Overall $73.5 \%$ of the children reported to have seen blood in their urine. Blood in urine was associated with $S$. haematobium infection categories. This remained in the final model, i.e., medium $(\mathrm{OR}=4.67, p<0.001)$ and high $(\mathrm{OR}=4.32, p<0.001)$.

One hundred and sixty-five (63.0\%) of the 262 children had macro-haematuria, and were infected with $S$. haematobium. Of the 97 children with no macro re-treated -haematuria, only 16 (17\%) were not infected with $S$. haematobium. Higher odds of macro-haematuria were associated with gender and age group. Multi-variable analysis showed that odds of finding macro-haematuria was higher in boys $(\mathrm{OR}=2.04, p<0.05)$ than girls, and those having medium $(\mathrm{OR}=10.6, p<0.001)$ and high $S$. haematobium intensity of infection ( $\mathrm{OR}=276.06, p<0.001)$ had higher odds of having macro-haematuria than those uninfected or with low intensity of $S$. haematobium infection.

The overall prevalence of micro-haematuria in the study population was $69 \%$. All children with microhaematuria were infected with $S$. haematobium and none of the uninfected children had micro-haematuria whereas $26 \%$ of infected children were negative for micro-haematuria. All children with high intensity of $S$. haematobium infection had micro-haematuria. This gave some problems in estimating coefficients in the logistic regression analysis. Therefore, for the multi-variable model medium and high intensity of S. haematobium were combined as one group. In the final model, the odds of children with medium and high intensity of $S$. haematobium having anemia was 46.13 (95 \% CL: 19.77-107.63; $\mathrm{p}<0.001)$ times that of children without or with light $S$. haematobium infection. In the same model children with medium/high intensity of A. lumbricoides infection had lower odds of anemia that children with no or light infection $(\mathrm{OR}=0.27,95 \% \mathrm{CL}$ : $0.11-0.64, p<0.01)$. 
White blood cell count was performed on blood samples from 217 children. The overall prevalence of eosinophilia (eosinophils $\geq 7 \%$ ) was $93 \%$. Odds of eosinophilia increased with hookworm infection intensity. None of the children had malaria parasites.

\section{Analysis of ultrasound detected morbidity}

Of the 262 children examined for S. haematobium infection, the urinary tracts of 219 (84 \%) were examined for morbidity using ultrasound (Table 2). Thirty-eight per cent of the children had different forms of urinary tract morbidity such as bladder wall thickness (31\%), bladder wall inner surface irregularity (30\%), bladder mass (7 \%), pseudopolyps (2\%), kidney pyelon dilatation (11\%) and ureter dilatation (4\%). The former four morbidity types were combined into lower urinary tract pathology and the latter two types were combined into upper urinary tract pathology.

The odds of bladder pathology increased with intensity of S. haematobium infection (OR $=2.85,95 \%$ CL: 1.32-6.19, $p<0.01$ and $\mathrm{OR}=4.40,95 \% \mathrm{CL}: p<0.001$ for medium and high intensity of infection, respectively). Multi-variable tests were not attempted due to the low number of cases with bladder pathology (Table 2).

Upper urinary tract pathology was associated significantly with high intensity of S. haematobium infection (Table 2) while total pathology did show significant association with $S$. haematobium infection and increased with intensity of $S$. haematobium infections.

Only 1 of the 16 children without S. haematobium infection had lower urinary tract pathology whereas 22 (11\%) of children with S. haematobium infection had upper urinary tract pathology and only one of those without the infection had upper urinary tract pathology.

\section{Discussion}

The present study analysed infection and morbidity due to S. haematobium and STH in primary school children from two neighbouring villages in northern coastal Kenya. In agreement with estimates for the general area [9], the prevalence and intensity of $S$. haematobium infections among the children were high suggesting intense transmission. This could partly be due to extensive seasonal floods, under-developed transport infrastructure and low hygiene standards in the area. For example, less than $5 \%$ of the households in the villages had latrines (data not shown) resulting in intense environmental contamination with helminth eggs. Children also extensively played in the nearby potentially snail-infested water bodies. Most infections with STH were of low intensity according to WHO classification [28]. Considering the low hygiene standards in the area, the reasons for this were not clear but could be due to harsh environmental conditions that did not favour survival and development of STH eggs and/or larval stages [14].

Only age group was found to be an important predictor for infection with $S$. haematobium among the children, with older children (10-12 years old) having higher odds than younger ones (5-9 years old). It is likely that older children had a higher tendency to get in contact with cercariae-infested water than younger children whose outdoor activities are more closely monitored and controlled by their parents. The finding that children in Ozi had higher odds of infection with hookworm than those in Kau suggests that different environmental factors influenced transmission in the two villages. It was observed that in Ozi, the soil was mainly sandy and covered with dense vegetation whereas in Kau the soil was mainly clay and sparsely covered with vegetation. These conditions are known to affect the survival and development of hookworm [35-38]. Boys in the two villages had a higher risk of infection with hookworm than girls suggesting that boys' behaviour exposed them to infective hookworm larvae more than girls as also seen elsewhere [39]. For example, during field data collection in the present study it was observed that most of the boys, but not girls, could have been exposed when playing football with bared feet on moist soils. The association of $T$. trichiura and A. lumbricoides infections to school, gender and age could be explained by the same environmental and behavioural differences given above with boys and older children being more exposed to the infections than girls and young children, respectively, due to their tendency to spend more time outdoors.

No significant association was observed between $S$. haematobium and STH. This agrees with the findings of a study of children in Pemba Island where no correlation of infection intensity between S. haematobium and STH (T. trichiura and A. lumbricoides) was seen [40]. The reason could be that $S$. haematobium and STH occupy different habitats in the human host and therefore may have minimal immunological interaction. It is also possible that the different modes of transmission of S. haematobium and STH may account for the lack of interaction [14].

Children with higher intensity of $T$. trichiura had higher odds of infection with hookworm and vice versa. The eggs and larvae of the two parasites have more or less similar environmental requirements and the observation in the present study could therefore be due to epidemiological coincidence [14]. However, this does not explain why a similar phenomenon was not observed between the two STH (hookworm and T. trichiura) and $A$. lumbricoides which have similar epidemiology. Experimental studies have suggested that intestinal nematodes, including hookworms and Trichuris spp., can bias the host immune responses to favour their survival and 
avoid expulsion from host [41, 42]. This might favour heterologous intestinal nematode infections, thus resulting in synergistic interactions between hookworm and T. trichiura. In agreement with this, high intensities of hookworm and $T$. trichiura infections were also positively associated with $A$. lumbricoides infection status although the interaction terms were not statistically significant.

Prevalences of low BMI, anaemia, macro-haematuria, micro-haematuria and eosinophilia were remarkably high among the children. Similarly high prevalences of micro- and macro-haematuria were previously among schoolchildren in Kilifi, another area of coastal Kenya [18]. Haematuria was associated with S. haematobium infections in agreement with the suggestion that haematuria among schoolchildren in coastal Kenya is mainly due to infections with this parasite [43].

The prevalence of ultrasound detectable pathology among the children was high and together with a report from older schoolchildren from Kilifi [18] these findings indicate that urinary tract pathology among children in coastal Kenya is mainly due to infection with the parasite. Considering that ultrasound detects longstanding/ chronic pathology $[44,45]$, the present study indicates that ultrasound-detectable pathology due to S. haematobium started to develop early in the lives of the children. Fortunately, late stage urinary tract morbidity such as hydronephrosis and hydroureter, which could potentially lead to renal failure, was not detected [46]. This suggests that treating children of this age group in the area to avert development of severe end-stage morbidity is important.

Infections with schistosomes and STH have been associated with poor nutritional status among children in endemic areas [47-50]. In the present study, higher BMI scores were associated with low hookworm intensity while lower scores were associated with high intensity of $S$. haematobium suggesting that infections with the two species played an important role in the nutritional status of the children. Although A. lumbricoides and T. trichiura have been associated with low BMI, a study in Ethiopia did not detect a significant association between the prevalence of STH and nutritional status [47, 48, 51]. In the present study there was no strong evidence to show that A. lumbricoides and T. trichiura contributed significantly to low BMI among the children probably because most of A. lumbricoides and T. trichiura infections were of low intensity $[27,28]$.

High intensities of S. haematobium and hookworm cause considerable blood loss and were associated with anaemia among the children [51-54]. Although T. trichiura and $A$. lumbricoides may cause anaemia $[51,53,55]$ their intensities were low and thus their effects may have been obscured by the effects of S. haematobium and hookworms in the present study.

\section{Conclusion}

The prevalence of S. haematobium and STH among primary school children in the study area was high with majority of S. haematobium infections being of high intensity whereas majority of STH infections were of low intensity. There was no evidence of association between S. haematobium and STH infections but hookworm and T. trichiura infections were positively associated. S. haematobium and hookworm were positively associated with anaemia and low BMI among the children. S. haematobium was moreover associated with early urinary tract morbidity, but late stage morbidity was not common. Increased coverage during mass administration of praziquantel and albendazole in the area is recommended to control morbidity due to these infections.

Competing interests

The authors declare that they have no competing interest.

\section{Authors' contributions}

KHN participated in designing the study, and coordinated fieldwork, laboratory analyses, data analysis, and writing of the manuscript. BJV participated in designing the study, and in data analysis and writing of the manuscript. PES participated in designing the study, and in field data collection, data analysis and writing of the manuscript. HM participated in data analyses. DAM participated in field data collection. GK participated in ultrasound examination of study subjects. WGJ participated in designing the study and reviewing the manuscript. BBE participated in designing the study, and in field data collection and writing of the manuscript. All the authors read and approved the final manuscript.

\section{Acknowledgements}

Our special thanks go to the children from Kau and Ozi Primary Schools for participation in the study, and to the parents and teachers for allowing us to carry it out. Special thanks are extended to Felistas W. Muthini and Patricia J. Korir (University of Nairobi), Patrick W. Kahora and Patrick Mburu (Mpeketoni sub-District Hospital) and George Simiyu (Ngao District Hospital) for technical assistance during specimen collection and examination, and Naomi W. Nderitu, (Nurse at Mpeketoni sub-District Hospital) and Edmund Ireri (Kenya Medical Research Institute) for drug administration and ultrasound examinations, respectively. Drivers Apollo Aloo and Gilbert Esiaranda (University of Nairobi) are thanked for their commitment and endurance to long driving hours on rough terrain. We also highly appreciate the efforts of Seth Rhova (Ngao District Hospital) and Muhamed Galana (Ozi Village) who tirelessly conducted community mobilization. The study received financial support from DBL-Centre for Health Research and Development, Denmark, through a PhD study grant to $\mathrm{KHN}$.

\section{Author details}

${ }^{1}$ Department of Medical Microbiology, College of Health Sciences, University of Nairobi, P.O. Box 19676-00202, Nairobi, Kenya. ²Department of Veterinary Disease Biology, Faculty of Health and Medical Sciences, University of Copenhagen, Dyrlægevej 100, 1870 Frederiksberg C, Denmark. ${ }^{3}$ Division of Vector Borne \& Neglected Tropical Diseases, Ministry of Public Health \& Sanitation, P.O. Box 54840-00202, Nairobi, Kenya. ${ }^{4}$ Centre for Biotechnology Research \& Development, Kenya Medical Research Institute, P. O. Box 54840-00200, Nairobi, Kenya. ${ }^{5}$ Jaramogi Oginga Odinga University of Science and Technology, P. O. Box 210-40601, Bondo, Kenya.

Received: 23 June 2015 Accepted: 27 January 2016

Published online: 03 February 2016

References

1. Pullan RL, Smith $J L$, Jasrasaria R, Brooker SJ. Global numbers of infection and disease burden of soil transmitted helminth infections in 2010. Parasit Vectors. 2014;7:37 
2. Colley DG. Morbidity control of schistosomiasis by mass drug administration: how can we do it best and what will it take to move on to elimination? Trop Med Health. 2014;42:S25-32.

3. WHO. Working to overcome the global impact of neglected tropical diseases: first WHO report on neglected tropical diseases. 2010.

4. Hotez PJ, Kamath A. Neglected tropical diseases in sub-saharan Africa: review of their prevalence, distribution, and disease burden. PLoS Negl Trop Dis. 2009;3:e412.

5. Pullan R, Brooker S. Impact of polyparasitism in humans : are we underestimating the burden of parasitic diseases ? Parasitology. 2009;135:783-94

6. Pullan RL, Bethony JM, Geiger SM, Cundill B, Correa-Oliveira R, Quinnell RJ, et al. Human helminth co-infection: analysis of spatial patterns and risk factors in a Brazilian community. PLoS Negl Trop Dis. 2008;2:e352.

7. Utzinger J, Raso G, Brooker S, De Savigny D, Tanner M, Ornbjerg N, et al. Schistosomiasis and neglected tropical diseases: towards integrated and sustainable control and a word of caution. Parasitology. 2009;136:1859-74.

8. Brooker S, Clements ACA. Spatial heterogeneity of parasite co-infection: determinants and geostatistical prediction at regional scales. Int J Parasitol. 2009;39:591-7.

9. Brooker S, Kabatereine NB, Smith JL, Mupfasoni D, Mwanje MT, Ndayishimiye O, et al. An updated atlas of human helminth infections: the example of East Africa. Int J Health Geogr. 2009;8:42.

10. Nagi S, Chadeka EA, Sunahara T, Mutungi F, Justin YKD, Kaneko S, et al. Risk factors and spatial distribution of Schistosoma mansoni infection among primary school children in Mbita District. Western Kenya PLoS Negl Trop Dis. 2014;8:e2991.

11. Geiger SM. Immuno-epidemiology of Schistosoma mansoni infections in endemic populations co-infected with soil-transmitted helminths: Present knowledge, challenges, and the need for further studies. Acta Trop. 2008:108:118-23.

12. Christensen NO, Nansen P, Fagbemi BO, Monrad J. Heterologous antagonistic and synergistic interactions between helminths and between helminths and protozoans in concurrent experimental infection of mammalian hosts. Parasitol Res. 1987:73:387-410.

13. Supali T, Verweij J, Wiria AE, Djuardi Y, Hamid F, Kaisar MMM, et al. Polyparasitism and its impact on the immune system. Int J Parasitol. 2010:40:1171-6.

14. Viney ME, Graham AL. Patterns and processes in parasite co-infection. Adv Parasitol. 2013:82:321-69.

15. Abruzzi A, Fried B. Coinfection of Schistosoma (Trematoda) with bacteria, protozoa and helminths. Adv Parasitol. 2011;77:1-85.

16. King $\mathrm{CH}$, Muchiri EM, Kariuki HC, Ouma JH, Mungai P, Kitron U, et al. Spatial patterns of urinary schistosomiasis infection in a highly endemic area of coastal Kenya. Am J Trop Med Hyg. 2004;70:443-8.

17. Mungai PL, Muchiri EM, King CH, Kitron U, Clennon JA. Spatial and temporal variations in local transmission of Schistosoma haematobium in Msambweni, Kenya. Am J Trop Med Hyg. 2006;75:1034-41.

18. Vennervald BJ, Kombe Y, Kihara RW, Ndzovu M, Mungai P, Ouma JH, et al. Parameters associated with Schistosoma haematobium infection before and after chemotherapy in school children from two villages in the coast province of Kenya. Trop Med Int Health. 1999;4:335-40

19. King $\mathrm{CH}$, Blanton RE, Muchiri EM, Ouma JH, Kariuki HC, Mungai P, et al. Low heritable component of risk for infection intensity and infection-associated disease in urinary schistosomiasis among Wadigo village populations in Coast Province, Kenya. Am J Trop Med Hyg. 2004;70:57-62.

20. Wamachi AN, Mayadev JS, Mungai PL, Magak PL, Ouma JH, Magambo JK, et al. Increased ratio of tumor necrosis factor - a to interleukin-10 production is associated with Schistosoma haematobium - induced urinary-tract morbidity. J Infect Dis. 2004;190:2020-30.

21. King CH. Long-term outcomes of school-based treatment for control of urinary schistosomiasis: a review of experience in coast province, Kenya. Mem Inst Oswaldo Cruz. 2006;101:299-306.

22. Magnussen P, Muchiri E, Mungai P, Ndzovu M, Ouma J, Tosha S. A schoolbased approach to the control of urinary schistosomiasis and intestinal helminth infections in children in Matuga, Kenya : impact of a 2 year chemotherapy programme on prevalence and intensity of infections. Trop Med Int Heal. 1997;2:825-31.

23. Njenga SM, Mutungi FM, Wamae CN, Mwanje MT, Njiru KK, Bockarie MJ. Once a year school-based deworming with praziquantel and Albendazole combination may not be adequate for control of urogenital schistosomiasis and hookworm infection in Matuga district, Kwale County. Kenya Parasit Vectors. 2014;7:74.
24. Sturrock HJW, Pullan RL, Kihara JH, Mwandawiro C, Brooker SJ. The use of bivariate spatial modeling of questionnaire and parasitology data to predict the distribution of Schistosoma haematobium in Coastal Kenya. PLoS Negl Trop Dis. 2013;7:e2016.

25. Njaanake KH, Simonsen PE, Vennervald BJ, Mukoko DA, Reimert CM, Gachuhi K et al. Urinary cytokines in Schistosoma haematobium- infected schoolchildren from Tana Delta District of Kenya. BMC Infect Dis. 2014;14:501.

26. Cheesbrough M. District laboratory practice in tropical countries part 1. Second Edi. Cambridge: Cambridge University Press; 2009.

27. WHO. Guidelines for the evaluation of soil-transmitted helminthiasis and schistomiasis at community level, vol. 1. 1998

28. Bergquist R, Johansen MV, Utzinger J. Diagnostic dilemmas in helminthology: what tools to use and when? Trends Parasitol. 2009:25:151-6.

29. Bench Aids for the diagnosis of intestinal parasites [http://apps.who.int/iris/ bitstream/10665/37323/1/9789241544764 eng.pdf?ua=1]

30. WHO. Worldwide prevalence of anaemia 1993-2005. Geneva: WHO Global Database on Anaemia; 2008

31. De Onis M, Habicht JP. Anthropometric reference data for international use: recommendations from a world health organization expert committee. Am J Clin Nutr. 1996;64:650-8.

32. WHO. Ultrasound in schistosomiasis: A practical guide to the standardized Use of ultrasonography for the assessment of schistosomiasis-related morbidity. Geneva: WHO; 2000

33. Hilbe JM. Negative Binomial Regression [Internet]. 2nd ed. Cambridge: Cambridge University Press; 2014 [cited 2015 May 26]. 572 p. Available from: https://books.google.com/books?id=kbihoAEACAAJ\&pgis=1.

34. Hilbe JM. Logistic Regression Models [Internet]. Cambridge: CRC Press; 2009 [cited 2015 May 26]. 656 p. Available from: https://books.google.com/ books?id=tmHMBQAAQBAJ\&pgis=1.

35. Mabaso MLH, Appleton CC, Hughes JC, Gouws E. The effect of soil type and climate on hookworm (Necator americanus) distribution in KwaZulu-Natal, South Africa. Trop Med Int Heal. 2003:8:722-7.

36. Weaver HJ, Hawdon JM, Hoberg EP. Soil-transmitted helminthiases : implications of climate change and human behavior. Trends Parasitol. 2010;26:574-81

37. Riess H, Clowes P, Kroidl I, Kowuor DO, Nsojo A, Mangu C, et al. Hookworm infection and environmental factors in Mbeya Region, Tanzania: A crosssectional, population-based study. PLoS Negl Trop Dis. 2013:7:e2408.

38. Ortega CD, Ogawa NY, Rocha MS, Blasbalg R, Caiado AHM, Warmbrand G, et al. Helminthic diseases in the abdomen: an epidemiologic and radiologic overview. Radiographics. 2010;30:253-68.

39. Tomono N, Anantaphruti MT, Jongsuksuntigul P, Thongthien P, Leerapan P, Silapharatsamee $Y$, et al. Risk factors of helminthiases among schoolchildren in southern Thailand. Southeast Asian J Trop Med Public Health. 2003:34:264-8.

40. Booth M, Mayombana C, Kilima P. The population biology and epidemiology of schistosome and geohelminth infections among schoolchildren in Tanzania. Trans R Soc Trop Med Hyg. 1998:92:491-5.

41. Gaze S, Bethony JM, Periago MV. Immunology of experimental and natural human hookworm infection. Parasite Immunol. 2014:36:358-66.

42. Klementowicz JE, Travis MA, Grencis RK. Trichuris muris: a model of gastrointestinal parasite infection. Semin Immunopathol. 2012;34:815-28.

43. Wamae CN, Lammie PJ. Haematuria in coastal Kenya is associated with Schistosoma haematobium but not Wuchereria bancrofti infection. Trans R Soc Trop Med Hyg. 1998;92:63-4.

44. Hatz C, Savioli L, Mayombana C, Dhunputh J, Kisumku UM, Tanner M. Measurement of schistosomiasis-related morbidity at community level in areas of different endemicity. Bull World Health Organ. 1990;68:777-87.

45. Vennervald BJ, Kahama Al, Reimert CM. Assessment of morbidity in Schistosoma haematobium infection : current methods and future tools. Acta Trop. 2000;77:81-9.

46. Hatz CF, Vennervald BJ, Nkulila T, Vounatsou P, Kombe Y, Mayombana C, et al. Evolution of Schistosoma haematobium-related pathology over 24 months after treatment with praziquantel among school children in southeastern Tanzania. Am J Trop Med Hyg. 1998:59:775-81.

47. Stephenson LS, Latham MC, Ottesen EA. Malnutrition and parasitic helminth infections. Parasitology. 2000;121(Suppl):S23-38.

48. Amare B, Ali J, Moges B, Yismaw G, Belyhun Y, Gebretsadik S, et al. Nutritional status, intestinal parasite infection and allergy among school children in northwest Ethiopia. BMC Pediatr. 2013;13:7.

49. Sanchez AL, Gabrie JA, Usuanlele M-T, Rueda MM, Canales M, Gyorkos TW. Soil-transmitted helminth infections and nutritional status in school- 
age children from rural communities in Honduras. PLoS Negl Trop Dis. 2013;7:e2378.

50. Kosinski KC, Adjei MN, Bosompem KM, Crocker JJ, Durant JL, Osabutey D, et al. Effective control of Schistosoma haematobium infection in a Ghanaian community following installation of a water recreation area. PLoS Negl Trop Dis. 2012;6:e1709.

51. Stephenson LS, Latham MC, Ottesen EA. Global malnutrition. Parasitology. 2000;121(Suppl):S5-S22.

52. Friedman JF, Kanzaria HK, McGarvey ST. Human schistosomiasis and anemia: the relationship and potential mechanisms. Trends Parasitol. 2005;21:386-92.

53. Khuroo MS, Khuroo MS, Khuroo NS. Trichuris dysentery syndrome: a common cause of chronic iron deficiency anemia in adults in an endemic area (with videos). Gastrointest Endosc. 2010;71:200-4.

54. Chen JM, Zhang XM, Wang $\sqcup$, Chen Y, Du Q, Cai JT. Overt gastrointestinal bleeding because of hookworm infection. Asian Pac J Trop Med. 2012:5:331-2.

55. Stephenson LS, Holland CV, Cooper ES. The public health significance of Trichuris trichiura. Parasitology. 2000;121:S73-95.

Submit your next manuscript to BioMed Central and we will help you at every step:

- We accept pre-submission inquiries

- Our selector tool helps you to find the most relevant journal

- We provide round the clock customer support

- Convenient online submission

- Thorough peer review

- Inclusion in PubMed and all major indexing services

- Maximum visibility for your research

Submit your manuscript at www.biomedcentral.com/submit
Biomed Central 